\title{
A PROPERTY OF HURWITZ POLYNOMIALS AND SOME ASSOCIATED INEQUALITIES FOR FOURIER TRANSFORMS
}

ARMEN H. ZEMANIAN

1. This paper considers functions, $Z(s)$, that are reciprocals of Hurwitz polynomials, $P(s)$, (those polynomials whose roots all have negative real parts) where $s$ is a complex variable equal to $\sigma+i t$. These functions are given by equations (1) and (2) and the coefficients, $b_{m-1}, b_{m-2}, \cdots, b_{0}$, are positive, real numbers $[3$, p. 395].

$$
\begin{aligned}
& Z(s)=\frac{1}{P(s)}, \\
& P(s)=s^{m}+b_{m-1} s^{m-1}+\cdots+b_{0} .
\end{aligned}
$$

In Theorem 1 it is shown that a function obtained by repeatedly integrating $Z(s)$ has either a real or an imaginary part which is of constant sign when $s$ is purely imaginary. Using this result, inequalities on the Fourier transforms of $Z(i t)$ are developed. The conclusions of Theorems 3 and 4 are generalizations of inequalities given in a previous paper [ 1 , Theorems 1,2 , and 3$]$. These in turn are somewhat similar to an inequality obtained by Boas and Kac [2].

2. The first result of this paper deals with the function $Z_{k}(s)$ which is derived from the function $Z(s)$ by successive integrations. This function is defined by (3) where the real parts of the complex variables $s_{0}, s_{1}, \cdots, s_{k-1}, s$ are all non-negative and $k \leqq m-1$.

$$
Z_{k}(s)=(-i)^{k} \int_{\infty}^{s} d s_{k-1} \int_{\infty}^{s_{k-1}} d s_{k-2} \cdots \int_{\infty}^{s_{1}} Z\left(s_{0}\right) d s_{0} .
$$

Letting $s_{k}=\sigma_{k}+i t_{k}$, the real and imaginary parts, $R_{k}(t)$ and $I_{k}(t)$, of $Z_{k}(i t)$ may be obtained from the real and imaginary parts, $R(t)$ and $I(t)$, of $Z(i t)$ by (4) and (5).

$$
\begin{aligned}
& R_{k}(t)=\int_{-\infty}^{t} d t_{k-1} \int_{-\infty}^{t_{k-1}} d t_{k-2} \cdots \int_{-\infty}^{t_{1}} R\left(t_{0}\right) d t_{0}, \\
& I_{k}(t)=\int_{-\infty}^{t} d t_{k-1} \int_{-\infty}^{t_{k-1}} d t_{k-2} \cdots \int_{-\infty}^{t_{1}} I\left(t_{0}\right) d t_{0} .
\end{aligned}
$$

Received by the editors October 1, 1956. 
That these integrations may be performed $(m-1)$ times will be shown subsequently.

The following well known property of Hurwitz polynomials [3, p. 400] will be used in the proof of Theorem 1.

Lemma 1. If $P(s)$ is a Hurwitz polynomial of mth degree, the even and odd parts of $P(s)$ are polynomials whose zeros are simple and lie on the it axis.

The following two simple lemmas will also be needed.

Lemma 2. Let $r(w)$ be even and integrable for $-\infty<w<\infty$; let $q(w)=\int_{-\infty}^{w} r(u) d u$, and let $q(\infty)=0$. Then $q(w)$ is odd.

Proof.

$$
q(\infty)=\int_{-\infty}^{w} r(u) d u+\int_{w}^{\infty} r(u) d u=0
$$

Therefore,

$$
q(w)=\int_{-\infty}^{w} r(u) d u=\int_{\infty}^{w} r(u) d u .
$$

Replacing $u$ in the last integral by $-v$ and setting $r(-v)=r(v)$ :

$$
q(w)=-\int_{-\infty}^{-w} r(v) d v=-q(-w) .
$$

Lemma 3. Let $p(w)$ be odd and integrable for $-\infty<w<\infty$; let $h(w)$ $=\int_{-\infty}^{w} p(u) d u$, and let $h(\infty)=0$. Then $h(w)$ is even.

This lemma may be proved in a similar fashion.

Theorem 1. Let $Z(s)$ be the reciprocal of a Hurwitz polynomial of mth degree as given by (1) and (2) and let $R_{k}(t)$ and $I_{k}(t)$ be defined by (4) and (5) where $k \leqq m-1$. Then $R_{k}(t)$ is even or odd and $I_{k}(t)$ is odd or even for $k$ even or odd respectively. Furthermore, for $m=2 n+1$ $(n=0,1,2, \cdots), R_{k}(t)$ has $m-1-k$ simple zeros, $I_{k}(t)$ has $m-k$ simple zeros and $(-1)^{n} R_{2 n}(t)>0$ for $-\infty<t<+\infty$; for $m=2 n$ $(n=1,2,3, \cdots), R_{k}(t)$ has $m-k$ simple zeros, $I_{k}(t)$ has $m-1-k$ simple zeros and $(-1)^{n+1} I_{2 n-1}(t)>0$ for $-\infty<t<+\infty$.

Proof. The $Z(i t)$ may be expressed as follows:

$$
Z(i t)=R(t)+i I(t)=\frac{P^{*}(i t)}{|P(i t)|^{2}}
$$

where $P^{*}(i t)$ is the complex conjugate of the Hurwitz polynomial 
for imaginary arguments. Since all the roots of $P(s)$ have negative real parts, $|P(i t)|^{2}$ is positive and finite for all finite $t$. Therefore by Lemma 1 , if $m$ is odd (or even), $R(t)$ will have $m-1$ (or $m$ ) simple zeros on the finite $t$ axis and $I(t)$ will have $m$ (or $m-1$ ) simple zeros on the finite $t$ axis.

Furthermore the inverse power series expansion of $Z(s)$, which holds for $|s| \geqq C$ where $C$ is greater than the distance from the origin to the pole of $Z(s)$ furthest way from the origin, is:

$$
Z(s)=\sum_{\nu=m}^{\infty} \frac{K_{\nu}}{s^{\nu}}
$$

where $K_{m}=1$. Since this series converges uniformly for $|s| \geqq C$, it may be integrated term by term yielding a resulting series which also converges uniformly in the same region. This process may be continued $k$ times where $k \leqq m-1 . Z_{k}(s)$ is defined by (3) and it may be expressed as follows

$$
Z_{k}(s)=i^{k} \sum_{\nu=m}^{\infty} \frac{K_{\nu}}{(\nu-1)(\nu-2) \cdots(\nu-k)} \cdot \frac{1}{s^{\nu-k}} .
$$

Since $Z(s)$ is analytic for $\sigma \geqq 0, Z_{k}(s)$ is also analytic in this region. Now consider a path of integration for the integral, $\Phi Z(s) d s$, which proceeds from $-i K$ up along the imaginary axis to $+i K$ and then continues in a semicircle through the $\sigma>0$ half plane back to $-i K$. Integrating $Z_{k}(s)$ around such a path, the contribution along the semicircular portion will vanish in the limit as $K$ goes to infinity if $k<m-1$ for then $Z_{k}(s)$ is $o(1 / s)$ as $s \rightarrow \infty$. Since $Z_{k}(s)$ is analytic in the $\sigma>0$ half plane, $R_{k+1}(\infty)=I_{k+1}(\infty)=0$.

First consider the case where $m=2 n+1(n=0,1,2, \cdots)$. The function, $R_{k+1}(t)$, must have less zeros in the interval, $-\infty<t \leqq \tau_{j}$, where $\tau_{j}$ is any zero of $R_{k}(t)$ than does $R_{k}(t)$. Moreover, invoking Lemmas 2 and 3 , it may be seen that $R_{k+1}(t)$ is odd or even when $R_{k}(t)$ is even or odd respectively so that $R_{k+1}(t)$ has less zeros in the interval, $\tau_{j} \leqq t<\infty$, than does $R_{k}(t)$. Thus each integration removes at least one zero from the finite $t$ axis. By the same argument each integration of $I(t)$ will remove at least one zero from the finite $t$ axis. Moreover $R(t)$ has $m-1$ simple zeros and $R_{m-1}(\infty)=0$, so that each integration must remove only one zero. (Otherwise $R_{m-1}(\infty)$ would not equal zero.) Therefore $R_{k}(t)$ has $m-1-k$ zeros and since $R(0)$ is positive, $(-1)^{n} R_{2 n}(t)>0$ for $-\infty<t<+\infty$. Also $I_{m-1}(t)$ is an odd function so that $I_{m}(\infty)=0$. Thus each integration of $I(t)$ will remove only one zero and $I_{k}(t)$ will have $m-k$ simple zeros for all 
finite real $t$.

The same argument given in the preceding paragraph may be applied, when $m=2 n(n=1,2,3, \cdots)$, to obtain the remaining portion of the conclusion of this theorem. In this case the roles of $R(t)$ and $I(t)$ are reversed and the fact that $d I / d t$ at $t=0$ is a negative quantity may be used to obtain the fact that $(-1)^{n+1} I_{2 n-1}(t)>0$ for $-\infty<t$ $<+\infty$.

3. The second part of this paper is concerned with the Fourier transform of $Z(i t)$ given by (7).

$$
f(x)=\frac{1}{2 \pi} \int_{-\infty}^{+\infty} Z(i t) e^{i x t} d t .
$$

Since $Z(s)$ has all its poles in the $\sigma<0$ half plane, $f(x)$ equals zero for $x<0$ and so $f(x)$ may be represented either by (8) or (9) for $x>0$.

$$
\begin{aligned}
& f(x)=\frac{2}{\pi} \int_{0}^{\infty} R(t) \cos x t d t . \\
& f(x)=-\frac{2}{\pi} \int_{0}^{\infty} I(t) \sin x t d t .
\end{aligned}
$$

THEOREM 2. If $Z(s)$ is the reciprocal of a Hurwitz polynomial of $m$ th degree as given by (1) and (2) and if $f(x)$ is defined by (7) then, for $0 \leqq x<\infty$,

$$
|f(x)| \leqq \frac{x^{m-1}}{(m-1) !} .
$$

PROOF. For $m=2 n+1(n=0,1,2, \cdots)$, upon integrating by parts expression (8) $2 n$ times and using the fact that $R_{k}(\infty)=0$ for $k<m$, the following may be obtained.

$$
f(x)=(-1)^{n} x^{2 n} \frac{2}{\pi} \int_{0}^{\infty} R_{2 n}(t) \cos x t d t .
$$

But $(-1)^{n} R_{2 n}(t)>0$ for all finite $t$ and so:

$$
|f(x)|<(-1)^{n} x^{2 n} \frac{2}{\pi} \int_{0}^{\infty} R_{2 n}(t) d t .
$$

By integrating $Z_{2 n}(s)$ around the $\sigma>0$ half plane, the integral, $\int_{0}^{\infty} R_{2 n}(t) d t$, is found to equal $(-1)^{n} \pi / 2(2 n)$ ! Thus the conclusion of this theorem is obtained for the case where $m$ is odd.

For $m=2 n$, a similar argument may be applied to (9). Integration by parts $(2 n-1)$ times yields: 


$$
f(x)=(-1)^{n+1} x^{2 n-1} \frac{2}{\pi} \int_{0}^{\infty} I_{2 n-1}(t) \cos x t d t .
$$

But by use of the first theorem,

$$
|f(x)| \leqq(-1)^{n+1} x^{2 n-1} \frac{2}{\pi} \int_{0}^{\infty} I_{2 n-1}(t) d t .
$$

Integrating $Z_{2 n-1}(s)$ around the $\sigma>0$ half plane, the integral on the right hand side of the last expression is found to be equal to $(-1)^{n+1} \pi / 2(2 n-1)$ ! This yields the conclusion once more.

It should be noted that the case where $m=1$ is trivial for then $f(x)=e^{-b_{0} x}$. If however $Z(s)$ is the ratio of two polynomials, $N(s) / P(s)$, whose leading coefficients are unity and where the degree of $P(s)$ is greater than the degree of $N(s)$ by $m$ and if $Z(s)$ satisfies the conclusions of Theorem 1 , then the result of Theorem 2 will apply to the Fourier transform of $Z(s)$ defined by (7), (8) and (9) and the case $m=1$ will not be trivial. This comment also applies to Theorems 3 and 4.

An inequality that the sine function satisfies will be used in the proofs of Theorems 3 and 4 . Its proof was given previously [ 1 , Lemma 3].

Lemma 4. For $0 \leqq y<1, x \geqq 0$ and $N$ a positive integer,

$$
\sin x \leqq Q_{0} x+\sum_{p=1}^{N} \frac{y Q_{2 p}}{p} \sin \frac{p x}{y}
$$

and

$$
\sin x \geqq-Q_{0} x+\sum_{p=1}^{N}(-1)^{p+1} \frac{y Q_{2 p}}{p} \sin \frac{p x}{y}
$$

where

$$
\begin{aligned}
Q_{0} & =1+\sum_{k=1}^{N} \frac{\left(-y^{2}\right)\left(1^{2}-y^{2}\right) \cdots\left[(k-1)^{2}-y^{2}\right]}{(k !)^{2}}, \\
Q_{2 p} & =(-1)^{p} 2 \sum_{k=p}^{N} \frac{\left(-y^{2}\right)\left(1^{2}-y^{2}\right) \cdots\left[(k-1)^{2}-y^{2}\right]}{(k-p) !(k+p) !} \\
p=1,2, \cdots, N . &
\end{aligned}
$$

The next theorem is in one sense a generalization of a result that has appeared previously [1, Theorems 1 and 2] where the conclusion of the following theorem has been shown to apply in the cases where 
$m=1$ and $m=2$ to the Fourier transforms of functions satisfying certain general conditions.

THEOREM 3. If $Z(s)$ is the reciprocal of a Hurwitz polynomial of mth degree as given by (1) and (2), if $f(x)$ is defined by (7) and if $|f(x)| \leqq A$ for $x \geqq 1$ where $A$ is a non-negative constant, then for $0 \leqq x<1$,

$$
|f(x)| \leqq \frac{x^{m-2} \sin \pi x}{\pi(m-1) !}+2 A x^{m} \frac{\sin \pi x}{\pi} \sum_{p=1}^{\infty} \frac{1}{p^{m-1}\left(p^{2}-x^{2}\right)} .
$$

Proof. First consider the case where $m=2 n+1(n=0,1,2, \cdots)$. Integrating ( 8 ) by parts $2 n-1$ times, (18) is obtained.

$$
f(x)=(-1)^{n-1} x^{2 n-1} \frac{2}{\pi} \int_{0}^{\infty} R_{2 n-1}(t) \sin x t d t .
$$

By Theorem 1, $(-1)^{n-1} R_{2 n-1}(t)$ is an odd function having one zero at the origin and is positive for $0<t<\infty$. The sine function may be replaced by the right hand side of (13) and the result integrated term by term to yield:

$$
f(x) \leqq(-1)^{n-1} x^{2 n} Q_{0} \frac{2}{\pi} \int_{0}^{\infty} t R_{2 n-1}(t) d t+\sum_{p=1}^{N}\left(\frac{y}{p}\right)^{2 n} Q_{2 p} f\left(\frac{p x}{y}\right) .
$$

Moreover integrating $Z_{m-1}(s)$ around the $\sigma>0$ half-plane, the value for $(2 \pi) \int_{0}^{\infty} t R_{m-2}(t) d t$ (which equals $\left.-(2 / \pi) \int_{0}^{\infty} R_{m-1}(t) d t\right)$ may be found to be $(-1)^{n+1} /(2 n)$ !. So:

$$
f(x) \leqq \frac{x^{2 n}}{(2 n) !} Q_{0}+\sum_{p=1}^{N}\left(\frac{y}{p}\right)^{2 n} Q_{2 p} f\left(\frac{p x}{y}\right) .
$$

From (8), it is evident that $|f(x)| \leqq(2 / \pi) \int_{0}^{\infty}|R(t)| d t$ so that the $f(x)$ are bounded for all $x$. Using this, it is found that the double series obtained by letting $N$ go to infinity in the right hand side of (19) converges absolutely for $0 \leqq y<1$. So summing over the $k$, the following may be obtained (for details, cf. [1]) where the $Q_{2 p}$ converge to the $q_{2 p}$ [4, Equations $2.8(46)$ and $\left.1.2(8)\right]$.

$$
f(x) \leqq \frac{x^{2 n}}{(2 n) !} q_{0}+\sum_{p=1}^{\infty}\left(\frac{y}{p}\right)^{2 n} q_{2 p} f\left(\frac{p x}{y}\right)
$$

where

$$
q_{0}=\frac{\sin \pi y}{\pi y}, \quad q_{2 p}=(-1)^{p+1} \frac{2 y \sin \pi y}{\pi\left(p^{2}-y^{2}\right)} .
$$

Now if $x / y$ is set equal to one and $f(p x / y)$ is replaced by $A$ for $p$ odd 
and by $-A$ for $p$ even, the upper bound of (17) is achieved. The lower bound is obtained similarly by use of (14) rather than (13).

The conclusion of this theorem may also be achieved in the case where $m=2 n(n=1,2,3, \cdots)$ by integrating (9) $2 n-2$ times and then proceeding in the same way.

Finally bounds on the function, $F(x)$, which is defined by (21) are given by the last theorem.

$$
F(x)=\int_{0}^{x} f(\xi) d \xi=\frac{2}{\pi} \int_{0}^{\infty} R(t) \frac{\sin x t}{t} d t, \quad x \geqq 0 .
$$

It is evident that $F(\infty)=R(0)=1 / b_{0}$.

THEOREM 4. If $Z(s)$ is the reciprocal of a Hurwitz polynomial of mth degree as given by (1) and (2), if $f(x)$ is defined by (7) and if $(1-\gamma) F(\infty) \leqq F(x) \leqq(1+\gamma) F(\infty)$ for $x \geqq 1$ where $F(x)$ is defined by (21) and $\gamma$ is a non-negative constant, then for $0 \leqq x<1$,

$$
\begin{aligned}
F(x) \leqq \frac{x^{m-1}}{\pi} \sin \pi x\left\{\frac{1}{m !}+2 F(\infty) x^{2}[\right. & \sum_{p=1}^{\infty} \frac{(-1)^{p+1}}{p^{m}\left(p^{2}-x^{2}\right)} \\
& \left.\left.+\gamma \sum_{p=1}^{\infty} \frac{1}{p^{m}\left(p^{2}-x^{2}\right)}\right]\right\},
\end{aligned}
$$

$$
F(x) \geqq-\frac{x^{m-1}}{\pi} \sin \pi x\left\{\frac{1}{m !}-2 F(\infty) x^{2}(1-\gamma) \sum_{p=1}^{\infty} \frac{1}{p^{m}\left(p^{2}-x^{2}\right)}\right\}
$$

Proof. To obtain the upper bound (22) for both cases where $m$ is even and $m$ is odd, the proof proceeds in the same way as in the preceding theorem until the inequality given by (19) is obtained where $2 n$ is replaced by $m-1$. Integrating according to (21),

$$
F(x) \leqq \frac{x^{m}}{m !} Q_{0}+\sum_{p=1}^{N}\left(\frac{y}{p}\right)^{m} Q_{2 F} F\left(\frac{p x}{y}\right), \quad x \geqq 0 .
$$

However $|F(x)| \leqq(2 x / \pi) \int_{0}^{\infty}|R(t)| d t$ for $x \geqq 0$. Thus $F(x)$ is bounded for $0 \leqq x<\infty$ and so it may be seen that the double series obtained by letting $N$ go to infinity in the right hand side of (24) converges absolutely. Upon letting $N$ go to infinity and summing over the $k$, (25) is obtained where the $q_{2 p}$ are given by (20).

$$
F(x) \leqq \frac{x^{m}}{m !} q_{0}+\sum_{p=1}^{\infty}\left(\frac{y}{p}\right)^{m} q_{2 p} F\left(\frac{p x}{y}\right) .
$$

Now setting $x / y$ equal to 1 and replacing $F(p x / y)$ by $(1+\gamma) F(\infty)$ for $p$ odd and by $(1-\gamma) F(\infty)$ for $p$ even, (22) is achieved. 
Expression (23) may be derived in a similar fashion using (14). In this case, the expression corresponding to (24) is the following.

$$
F(x) \geqq-\frac{x^{m}}{m !} Q_{0}+\sum_{p=1}^{N}(-1)^{p+1}\left(\frac{y}{p}\right)^{m} Q_{2 p} F\left(\frac{p x}{y}\right) .
$$

As $N$ goes to infinity the double series converges absolutely again. Also the $|F(p x / y)|$ must be replaced by $(1-\gamma) F(\infty)$ for all $p$ to maintain the inequality.

\section{BIBLIOGRAPHY}

1. A. H. Zemanian, Some inequalities for Fourier transforms, Proc. Amer. Math. Soc. vol. 8 (1957) pp. 468-475.

2. R. P. Boas, Jr. and M. Kac, Inequalities for Fourier transforms of positive functions, Duke Math. J. vol. 12 (1945) pp. 189-206.

3. E. A. Guillemin, The mathematics of circuit analysis, New York, John Wiley and Sons, 1949.

4. A. Erdélyi, W. Magnus, F. Oberhettinger, and F. G. Tricomi, Higher transcendental functions, vol. I, New York, McGraw-Hill, 1953.

New York University 